\title{
Interface resistance of disordered magnetic multilayers
}

\author{
K. Xia ${ }^{1}$, P. J. Kelly ${ }^{1}$, G. E. W. Bauer ${ }^{2}$, I. Turek ${ }^{3}$, J. Kudrnovský ${ }^{4}$, and V. Drchal ${ }^{4}$ \\ ${ }^{1}$ Faculty of Applied Physics and MESA ${ }^{+}$Research Institute, University of Twente, \\ P.O. Box 217, 7500 AE Enschede, The Netherlands \\ ${ }^{2}$ Department of Applied Physics and DIMES, Delft University of Technology, \\ Lorentzweg 1, 2628 CJ Delft, The Netherlands \\ ${ }^{3}$ Institute of Physics of Materials, Academy of Sciences of the Czech Republic, CZ-616 62 Brno, Czech Republic \\ ${ }^{4}$ Institute of Physics, Academy of Sciences of the Czech Republic, CZ-182 21 Prague, Czech Republic
}

(November 8, 2018)

We study the effect of interface disorder on the spindependent interface resistances of $\mathrm{Co} / \mathrm{Cu}, \mathrm{Fe} / \mathrm{Cr}$ and $\mathrm{Au} / \mathrm{Ag}$ multilayers using a newly developed method for calculating transmission matrices from first-principles. The efficient implementation using tight-binding linear-muffin-tin orbitals allows us to model interface disorder using large lateral supercells whereby specular and diffuse scattering are treated on an equal footing. Without introducing any free parameters, quantitative agreement with experiment is obtained. We predict that disorder reduces the majority-spin interface resistance of $\mathrm{Fe} / \mathrm{Cr}(100)$ multilayers by a factor 3 .

When two layers of magnetic material are separated by a non-magnetic spacer layer, the electrical resistance of the system depends strongly on whether the magnetization directions are aligned parallel or anti-parallel. This effect is known as giant magnetoresistance (GMR) [1]. The huge interest [2 24 in the physics of GMR is largely driven by the wide application potential of the effect, which has already been realized in magnetic recording heads.

GMR can be observed in a number of different measuring configurations. The current-in-plane (CIP) configuration is experimentally the simplest and is what is used at present in applications. However, for gaining a better understanding of the underlying physics, the current-perpendicular-to-the-plane (CPP) configuration 3,5, 8 is preferred because of its higher symmetry which should make it easier to understand and because of higher MR ratios.

The factors usually considered in theoretical treatments of GMR are the potential steps encountered by electrons passing from one material to another, impurity scattering in the bulk of the layers, and defect scattering at the interfaces 24. There has been a great deal of discussion about the relative importance of these ingredients and their spin dependence, which cannot be resolved solely on the basis of model calculations which include these effects in parameterized form. Once the question has been suitably posed, however, detailed electronic structure calculations can be used to resolve the issue quantitatively. For example, the effect of potential steps and their microscopic origin could be established in this way [9,10].

In this Letter we wish to address the relative role of specular and diffuse interface scattering. This has been studied by a large number of authors but so far only using simple models which do not allow for detailed quantitative analysis of specific materials 11,12 . We focus on the interface resistance of the resistor model which describes the observed thickness and layer dependence of CPP-GMR remarkably well [3]. Because it turns out to be strongly spin-dependent and dominates the magnetoresistance for layer thicknesses which are not too large, the key to understanding CPP magnetoresistance lies in understanding the origin of the interface resistance. The methodology which we have developed allows us to include specular and diffuse scattering on an equal footing without introducing any arbitrary fitting parameters.

Explicit expressions for the interface resistance were derived by Schep et al. [13] in terms of the transmission matrix $T$ which describes how the electronic structure mismatch at an $A / B$ interface affects electron transport. In the limit in which there is no coherent scattering between adjacent interfaces, presumably due to sufficiently strong bulk scattering, the interface resistance is given by

$$
R_{A / B}=\frac{h}{e^{2}}\left[\frac{1}{\sum T_{\mu \nu}}-\frac{1}{2}\left(\frac{1}{N_{A}}+\frac{1}{N_{B}}\right)\right] .
$$

where $T_{\mu \nu}$ are the probabilities for eigenstate $\mu$ in material $A$ to be transmitted through the interface into the eigenstate $\nu$ in material $B$, the sum is over the Fermi surface, and $e^{2} / h N_{A(B)}$ is the Sharvin conductance of material $\mathrm{A}(\mathrm{B})$. In Ref. [13] transmission matrices and interface resistances were obtained for ideal $\mathrm{Co} / \mathrm{Cu}$ interfaces using a first-principles FLAPW based embedding technique. These, and similar results obtained by Stiles and Penn [14, demonstrated that a combination of spin-independent bulk scattering and strongly spindependent specular interface scattering arising from the spin-dependence of the band mismatch can account for the observed spin-dependence of the interface resistances. These results are at odds with the common wisdom that metallic heterointerfaces cannot be perfect due to unavoidable roughness and/or interface alloying. Indeed, 
for the one case of $\mathrm{Co} / \mathrm{Cu}(111)$ interfaces for which direct comparison could be made with experiment, the agreement though reasonable, was not perfect. We therefore address the following questions: Why does a calculation for a perfect interface agrees so well as it does for a sample produced by sputtering? Can theory and experiment be brought to even better agreement by taking into account disorder? Is the finding that specular interfaces are a reasonable first order approximation generic or a coincidence found only for the $\mathrm{Co} / \mathrm{Cu}$ system?

The FLAPW-based method used in Ref. [13] was computationally too demanding to allow interface disorder to be treated. Starting instead with the more efficient surface Green's function method [15] implemented with a tight-binding linear muffin tin orbital basis [16], we can now calculate the transmission and reflection matrices needed in the Landauer-Büttiker formulation of transport theory [17], but now for much larger systems. In this Letter, we present the results of calculations for $\mathrm{Co} / \mathrm{Cu}$, $\mathrm{Fe} / \mathrm{Cr}$ and $\mathrm{Ag} / \mathrm{Au}$ layered systems in which we model interface disorder by means of large lateral supercells. The electronic structure is determined self-consistently within the local spin density approximation. To model the interface, we randomly distribute the appropriate concentration of different atoms within lateral supercells 18 containing as many as $10 \times 10$ atoms. For the disordered layers the potentials are determined self-consistently using the layer CPA approximation 15]. The calculations are carried out with a $\mathbf{k}_{\|}$mesh density equivalent to 3600 $\mathbf{k}_{\|}$mesh points in the two dimensional Brillouin zone (BZ) of a $1 \times 1$ interface unit cell. The numerical error bar resulting from this sampling is smaller than $0.2 \%$ of the conductance. The interface resistances calculated for $\mathrm{Co} / \mathrm{Cu}$ (100) and (111) in the clean limit using Eq. (11) agree with those obtained by Schep using an entirely different code to within about $0.1 \mathrm{f} \Omega \mathrm{m}^{2}$ or $5 \%$.

In the presence of defects, the conductance can be expressed as the sum of a ballistic part and a diffuse part; the transmission matrix elements between two Bloch states with the same $\mathbf{k}_{\|}$correspond to ballistic scattering, those between two Bloch states with different $\mathbf{k}_{\|}$to diffuse scattering.

TABLE I. Results of calculations

\begin{tabular}{|c|c|c|c|}
\hline system & roughness & $R_{\text {maj }}\left(f \Omega m^{2}\right)$ & $\mathrm{R}_{\min }\left(\mathrm{f} \Omega \mathrm{m}^{2}\right)$ \\
\hline $\mathrm{Au} / \mathrm{Ag}(111)$ & clean & 0.094 & 0.094 \\
\hline $\mathrm{Au} / \mathrm{Ag}(111)$ & 2 layers 50-50 alloy & 0.118 & 0.118 \\
\hline $\mathrm{Au} / \mathrm{Ag}(111)$ & exp. & $0.100 \pm 0.008$ & $0.100 \pm 0.00$ \\
\hline $\mathrm{Co} / \mathrm{Cu}(100)$ & clean & 0.33 & 1.79 \\
\hline $\mathrm{Co}_{h c p} / \mathrm{Cu}(111)$ & clean & 0.60 & 2.24 \\
\hline $\mathrm{Co} / \mathrm{Cu}(111)$ & clean & 0.39 & 1.46 \\
\hline $\mathrm{Co} / \mathrm{Cu}(111)$ & 2 layers 50-50 alloy & 0.41 & $1.82 \pm 0.03$ \\
\hline $\mathrm{Co} / \mathrm{Cu}(111)$ & exp. & $0.26 \pm 0.06$ & $1.84 \pm 0.14$ \\
\hline $\mathrm{Fe} / \mathrm{Cr}(100)$ & clean & 2.82 & 0.50 \\
\hline $\mathrm{Fe} / \mathrm{Cr}(100)$ & 2 layers 50-50 alloy & 0.99 & 0.50 \\
\hline
\end{tabular}

The calculated results are shown in Table [. The $\mathrm{Au} / \mathrm{Ag}$ interface has fcc(111) texture and the interface roughness is estimated to be at least two layers thick in the MSU samples [19]. This makes it very suitable for testing our method without the complicating factor of spin dependence. The interface resistance we find for a clean $\mathrm{Au} / \mathrm{Ag}$ interface based on Eq. (11) is $0.047 \mathrm{f} \Omega \mathrm{m}^{2}$ which is very close to the experimental value, $0.050 \pm 0.004 \mathrm{f} \Omega \mathrm{m}^{2}$. The resistance of a $\mathrm{Au} / \mathrm{Ag}$ interface becomes $0.059 \mathrm{f} \Omega \mathrm{m}^{2}$ when the interface contains two layers of $\mathrm{Au}_{0.5} \mathrm{Ag}_{0.5}$ alloy. The uncertainty arising from using different alloy configurations within the $6 \times 6$ unit cell is less than $1 \%$.

We calculate the interface resistance of $\mathrm{Co} / \mathrm{Cu}$ interfaces for both (100) and (111) orientations. The lattice constant used for fcc $\mathrm{Co} / \mathrm{Cu}$ is $3.549 \AA$. We focus mainly on the (111) orientation as this is the structure which is predominantly seen in the experimental samples. The interface alloy is again at least two atomic layers thick [8]. We treat the interface disorder as two layers of $\mathrm{CoCu}$ alloy modelled using an $8 \times 8$ lateral supercell. The largest uncertainty between different configurations of two layers of 50-50 alloy is about $2.5 \%$, which is much smaller than the experimental error bar. For interface alloy compositions ranging from 50-50 to 44-56 the interface resistance does not change within our numerical accuracy. With two layers of interface alloy, the calculated transmission probability for the minority spin electrons decreases by about $10 \%$ bringing the calculated interface resistance into near perfect agreement with experiment. We find that disorder gives rise to mainly forward scattering of the electrons so that the decrease of the ballistic component is almost cancelled by the increase of the diffusive part. This is the reason why the calculations for the defect-free $\mathrm{Co} / \mathrm{Cu}$ interface [13, 14] were in reasonable agreement with experiment. The strong diffuse scattering also explains why the two-channel resistor model performs so well down to relatively thin layers in which bulk scattering should not be important.

The resistance of a $\mathrm{Cu} / \mathrm{Co}$ interface calculated with two layers of $50 \%$ interface alloy is $0.41 \mathrm{f} \Omega \mathrm{m}^{2}$ for the majority spin and $1.82 \pm 0.03 \mathrm{f} \Omega \mathrm{m}^{2}$ for the minority spin. The error bar for the minority-spin results from using a finite lateral supercell for modelling disorder and configuration averaging. The majority spin bands of $\mathrm{Cu}$ and $\mathrm{Co}$, like the bands of $\mathrm{Au}$ and $\mathrm{Ag}$, are so well matched that interface disorder has very little effect on the interface resistance. We observe (Table I) that there is near perfect agreement for the minority spin (certainly within the overall uncertainty of the calculation) but that the calculated resistance for the majority spin case which was already too large in the absence of disorder is even slightly increased by disorder. 


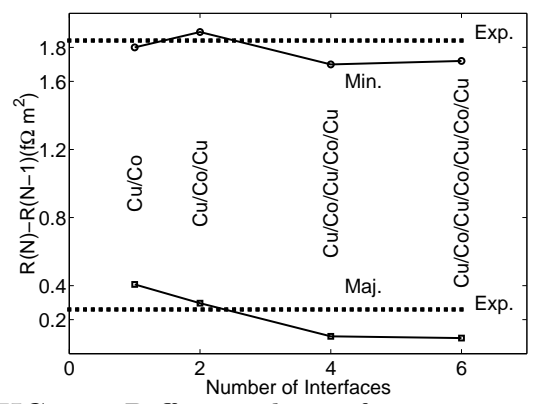

FIG. 1. Differential interface resistance as the number of interfaces increase for a disordered $\mathrm{Cu} / \mathrm{Co}$ multilayer embedded between $\mathrm{Cu}$ leads.

With two layers of interface alloy, almost $80 \%$ of the minority spin current results from diffuse scattering. We can see this in a different way by calculating the conductance for $\mathrm{Cu} / \mathrm{Co} / \mathrm{Cu}, \mathrm{Cu} / \mathrm{Co} / \mathrm{Cu} / \mathrm{Co} / \mathrm{Cu}$, and $\mathrm{Cu} / \mathrm{Co} / \mathrm{Cu} / \mathrm{Co} / \mathrm{Cu} / \mathrm{Co} / \mathrm{Cu}$ in a manner quite analogous to that described above for the single $\mathrm{Cu} / \mathrm{Co}$ interface. To be able to perform this large calculation we had to use a smaller supercell $(6 \times 6)$ so that the error bar is larger than for the $8 \times 8$ calculation. In these calculations the boundary $\mathrm{Cu}$ layers are semiinfinite "leads" and the interface disorder is two layers of 50-50 alloy. Results are insensitive to the individial layer thicknesses, chosen here to be 10 atomic layers. In Fig. 1 the differential resistance per interface is shown for all four systems. For the minority spins, the deviation from the result obtained for a single interface is quite small. The strong diffuse interface scattering destroys the phase coherent scattering between subsequent interfaces so that Ohm's law holds when the number of interfaces is increased. For the majority spin, however, the interface resistance does not obey Ohm's law but decreases as the number of interfaces increases. It appears to saturate at a value of $0.07 \mathrm{f} \Omega \mathrm{m}^{2}$ which is only a third of the experimental value, but consistent with Mathon's calculation for a multilayer with random layer thicknesses 20]. The majority spin potentials are so similar that the scattering from a double alloy layer is insufficient to break the coherence which is considerably longer range than for the strongly scattered minority spins. We would have to assume that the majority spin electrons remain coherent for transport through 4 interfaces in order to obtain a value of the interface resistance close to the experimental value of $0.26 \mathrm{f} \Omega \mathrm{m}^{2}$. Compared to real samples with bulk defects and lateral variations in the layer thicknesses, it appears that we overestimate

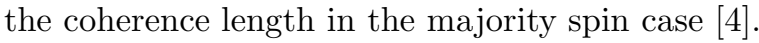

For the (111) orientation we also considered an interface between hcp $\mathrm{Co}$ and fcc $\mathrm{Cu}$. For a clean $\mathrm{Co}_{\mathrm{hcp}}^{(0001)} / \mathrm{Cu}_{\mathrm{fcc}}^{(111)}$ interface both majority and minority spin resistances are substantially larger than for the fcc case and even larger than the experimental values (Table II). In view of the substantial difference predicted between the two, it should be interesting to try and mea- sure it.
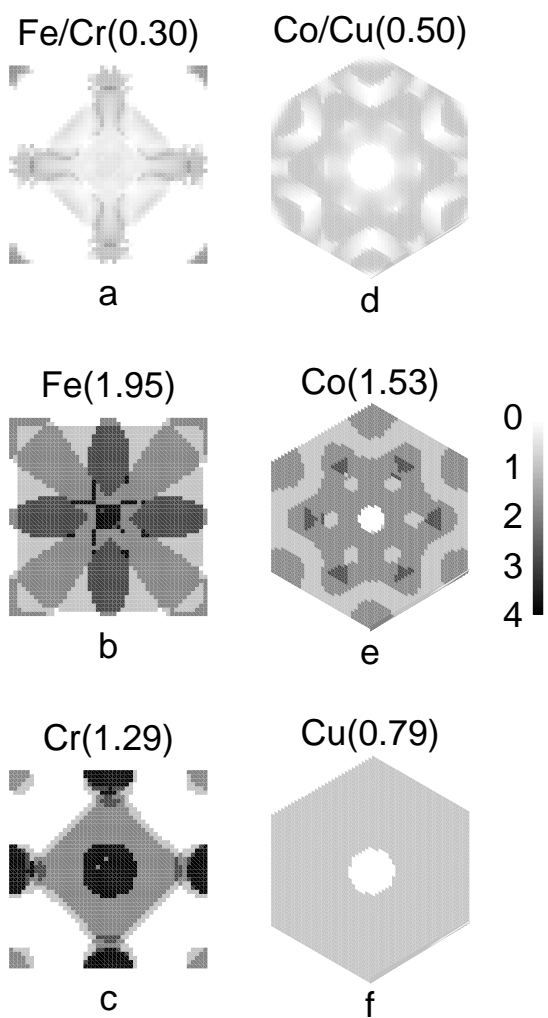

FIG. 2. Number of propagating channels in first Brillouin Zone. (a), (b) and (c) are for the majority spin of a clean $\mathrm{Fe} / \mathrm{Cr}$ interface, bulk Fe, and bulk $\mathrm{Cr}$,(d), (e) and (f) are for minority spin electrons of a clean $\mathrm{Co} / \mathrm{Cu}$ interface, bulk $\mathrm{Co}$, and bulk $\mathrm{Cu}$, respectively. The total number of propagating channels per unit cell is given in brackets on top of the figures. The grayscale interpolates the number of propagating channels per $k_{\|}$-point between zero (white) and four (black).

The $\mathrm{Fe} / \mathrm{Cr}$ (non-magnetic) interface calculation is performed for the bcc(100) orientation, which is the low index orientation with the largest spin-asymmetry 14. We used a lattice constant of $2.87 \AA$. To model the interface roughness a $6 \times 6$ lateral supercell was used. The uncertainty from configuration averaging is less than $10 \%$.

Whereas for $\mathrm{Co} / \mathrm{Cu}$ the majority-spin band structures were well matched, for $\mathrm{Fe} / \mathrm{Cr}$ the situation is reversed and it is the minority-spin electronic structures which match well. Using Eq. (11), the interface resistance is $2.82 \mathrm{f} \Omega \mathrm{m}^{2}$ and $0.50 \mathrm{f} \Omega \mathrm{m}^{2}$ for majority and minority spin, respectively, so that the $\mathrm{Fe} / \mathrm{Cr}$ interface has a negative spin-asymmetry, opposite to that of bulk Fe. The effect of disorder is to suppress the interface asymmetry rather than enhance it. As was the case for the $\mathrm{Co} / \mathrm{Cu}$ 
majority spins, interface disorder has only a small effect on the well-matched minority spin channel. For the majority spin channel however, the transmission probability for a clean interface is very low due to a large band mismatch. For a disordered interface, the ballistic contribution to the conductance can only decrease by a small amount but the diffuse component increases enormously leading to a large net increase in the transmission. $3 \%$ Fe impurities in the first $\mathrm{Cr}$ layer (or $3 \% \mathrm{Cr}$ in the first Fe layer) increase the transmission probability by more than 10\%. Two interdiffused atom layers suppress the spin asymmetry and the MR efficiently - the interface resistances resulting from two 50-50 interface alloy layers are $0.99 \mathrm{f} \Omega \mathrm{m}^{2}$ and $0.50 \mathrm{f} \Omega \mathrm{m}^{2}$ for majority and minority spin respectively. Vice versa, the interface quality is much more critical for a large CPP-MR in the $\mathrm{Fe} / \mathrm{Cr}$ than in the $\mathrm{Co} / \mathrm{Cu}$ system.

The qualitative difference between $\mathrm{Fe} / \mathrm{Cr}$ and $\mathrm{Co} / \mathrm{Cu}$ can be understood using Figs. 2(a-c) and Figs. 2(df) where we show as a function of $\mathbf{k}_{\|}$the number of majority-spin propagating channels for the $\mathrm{Fe} / \mathrm{Cr}$ interface, bulk Fe, and bulk $\mathrm{Cr}$, respectively, and the number of minority-spin propagating channels for the $\mathrm{Co} / \mathrm{Cu}$ interface, bulk $\mathrm{Co}$, and bulk $\mathrm{Cu}$, all in the first BZ. For the $\mathrm{Co} / \mathrm{Cu}$ (111) interface minority-spin states, the Fermi surfaces of both $\mathrm{Co}$ and $\mathrm{Cu}$ occupy a large part of the 2D BZ so that there are a large number of states with the same $\mathbf{k}_{\|}$in both materials which can, in principle, propagate in the absence of disorder. Summing over all $\mathbf{k}_{\|}$, however, the transmission probability of states (coming from $\mathrm{Cu}$ ) is only about $60 \%$; the character of the bulk states on either side is such that they match poorly. Defect scattering tends to reduce the transmission probability and thus increases the interface resistance of the $\mathrm{Co} / \mathrm{Cu}$ minority spin channel. On the other hand, we can identify two mechanisms by which interface disorder decreases the interface resistance in the $\mathrm{Fe} / \mathrm{Cr}$ majorityspin channel by a factor 3. Majority spin electrons with small $\mathbf{k}_{\|}$are almost completely reflected at the $\mathrm{Fe} / \mathrm{Cr}$ specular interface because the electronic states on both sides of the interface do not match well. Defect scattering is found to increase the transmission of these electrons strongly. Furthermore, for $\mathbf{k}_{\|}$outside of this central area, there are no propagating states on the $\mathrm{Cr}$ side. Propagating modes in Fe with larger $\mathbf{k}_{\|}$, which are totally reflected at the specular interface, can be scattered diffusely into the center of the BZ where many states are available in Cr.

In summary, we have studied the interface resistance of $\mathrm{Co} / \mathrm{Cu}, \mathrm{Fe} / \mathrm{Cr}$ and $\mathrm{Au} / \mathrm{Ag}$ interfaces. Depending on the system, interface disorder can increase or decrease the interface resistance. For some interfaces such as $\mathrm{Au} / \mathrm{Ag}$ and $\mathrm{Co} / \mathrm{Cu}$, the band mismatch at an interface is responsible for most of the interface resistance. For other systems such as $\mathrm{Fe} / \mathrm{Cr}$, the interface resistance can dramatically depend on the interface perfection. For Fe/Cr in- terface, the majority-spin interface resistance is reduced as much as $70 \%$ by interface disorder. Interface disorder enhances the spin asymmetry in the $\mathrm{Co} / \mathrm{Cu}$ system but decreases it for $\mathrm{Fe} / \mathrm{Cr}$. In the systems considered, the diffuse scattering arising from interface disorder breaks the phase coherence in high resistance spin channels, but not necessarily for the low resistance spin channels.

This work is part of the research program for the "Stichting voor Fundamenteel Onderzoek der Materie" (FOM), which is financially supported by the "Nederlandse Organisatie voor Wetenschappelijk Onderzoek" (NWO). This study was supported by the NEDO joint research program (NTDP-98), the Grant Agency of the Czech Republic (202/00/0122), and the Grant Agency of the Academy of Sciences of the Czech Republic (A1010829). We acknowledge benefits from the TMR Research Network on "Interface Magnetism" under contract No. FMRX-CT96-0089 (DG12-MIHT).

[1] M.N. Baibich et al., Phys. Rev. Lett. 61, 2472 (1988); G. Binasch et al., Phys. Rev. B 39, 4828 (1989).

[2] P.M. Levy, Solid State Phys. 47, 367 (1994).

[3] M.A.M. Gijs and G.E.W. Bauer, Advances in Physics 46, 285 (1997); J.-P. Ansermet, J. Phys.-Cond Mat. 10, 6027 (1998).

[4] A. Barthélémy, A. Fert and F. Petroff, Handbook of magnetic materials, Vol. 12, edited by K.H.J. Buschow (1999)

[5] W.P. Pratt, Jr., S.-F. Lee, J.M. Slaughter, R. Loloee, P.A. Schroeder, and J. Bass, Phys. Rev. Lett. 66, 3060 (1991).

[6] M.A.M. Gijs, S.K.J. Lenczowski, and J.B. Giesbers, Phys. Rev. Lett. 70, 3343 (1993).

[7] T.Valet and A. Fert, Phys. Rev. B48, 7099(1993).

[8] J. Bass and W.P. Pratt Jr., J. Magn. Magn. Mater. 200, $274(1999)$

[9] K.M. Schep, P.J. Kelly, and G.E.W. Bauer, Phys. Rev. Lett. 74, 586 (1995).

[10] P. Zahn, I. Mertig, M. Richter, and H. Eschrig, Phys. Rev. Lett. 75, 2996 (1995).

[11] A. Brataas and G.E.W. Bauer, Phys. Rev. B49, 14684(1994)

[12] S. Zhang and P.M. Levy, Phys. Rev. B57, 5336(1998)

[13] K.M. Schep, J.B.A.N. van Hoof, P.J. Kelly, G.E.W. Bauer, and J.E. Inglesfield, Phys. Rev. B56, 10805(1997)

[14] M. D. Stiles and D. R. Penn, Phys. Rev. B61, 3200 (2000).

[15] I. Turek, V. Drchal, J. Kudrnovský, M. Šob, and P. Weinberger, Electronic Structure of Disordered Alloys, Surfaces and Interfaces (Kluwer, Boston-London-Dordrecht, 1997)

[16] O. K. Andersen, O. Jepsen, and D. Glötzel, in Highlights in Condensed Matter Theory, edited by F. Bassani, F. Fumi and M. P. Tosi (North-Holland, Amsterdam, 1985), p. 59 . 
[17] S. Datta, Electronic Transport in Mesoscopic Systems (Cambridge University Press, Cambridge, 1995).

[18] P. Bruno et al., J. Mag. Mag. Mat. 198-199, 46 (1999); J. Kudrnovský et al., Czech. J. Phys. 49, 1583 (1999).

[19] L.L. Henry, Q. Yand, W.-C. Chiang, P. Holody, R. Loloee, W.P. Pratt, Jr., and J. Bass, Phys. Rev. B54, 12336 (1996)

[20] J. Mathon, Phys. Rev. B. 55, 960 (1997). 